\title{
Efectos de una terapia sistémica con azitromicina en el tratamiento de la periodontitis crónica
}

\author{
PLAZA JC * \\ GALLARDO $\mathbf{F} * *$ \\ DAVILA L $\mathbf{L} * *$ \\ RIOSECO $\mathbf{M} * * * *$
}

\author{
Plaza JC, Gallardo F, Dávila L, Rioseco M. Efectos de una terapia \\ sistémica con azitromicina en el tratamiento de la periodontitis \\ crónica. Av Periodon Implantol. 2003; 15, 1:35-42.
}

\begin{abstract}
RESUIMEN
Este estudio se efectuó con el objetivo fundamental de evaluar la eficacia de la administración sistémica de azitromicina como complemento a la terapia mecánica convencional en un grupo de pacientes con periodontitis crónica. El diseño del estudio fue prospectivo, controlado, randomizado y doble ciego. La muestra total correspondió a 19 pacientes con diagnóstico clínico de periodontitis crónica. Se realizaron mediciones clínicas para estudiar los siguientes parámetros: Indice Gingival de Loe y Silness, Indice de Placa de Silness y Loe, presencia de sangramiento al sondaje, profundidad de los sacos y niveles de inserción relativa en los días 0,30 y 60 . El total de pacientes, se distribuyó al azar, de manera que 10 pacientes recibieron azitromicina (500 mg cada 24 horas durante 3 días) y 9 recibieron el placebo. Todos los pacientes que participaron recibieron al inicio del tratamiento una higienización supragingival de toda la boca mediante ultrasonido, luego se tomaron impresiones del maxilar que correspondía según la ubicación de las piezas dentarias seleccionadas para la confección de las canaletas de acrílico que sirvieron de punto de referencia fijo para las mediciones de los niveles de inserción y profundidades de sacos. De los dientes seleccionados en el mismo maxilar, 2 fueron tratadas mediante raspado y pulido radicular, junto a sus vecinas inmediatas, mientras que las 2 remanentes permanecieron como control. Así se conformaron los 4 grupos de tratamiento: azitromicina, azitromicina + raspado y pulido radicular, placebo y placebo + raspado y pulido radicular. Todos los pacientes fueron controlados en los días 0,30 y 60 , siendo el día 0 el momento en que se efectuaron las mediciones de los parámetros clínicos mencionados, y luego se evaluaron a los 30 y 60 días. A los 30 días de control, la azitromicina redujo significativamente los niveles de inflamación periodontal y la profundidad de los sacos, en comparación con los valores basales, y asociada al raspado y pulido radicular mejoró significativamente los niveles de inserción, aunque la comparación entre los tratamientos demostró una superioridad del antibiótico sólo en el parámetro Indice Gingival
\end{abstract}

Nuestros resultados obtenidos en una muestra de 19 pacientes, que fueron controlados a los 30 días sugieren que la azitromicina asociada a raspado y pulido radicular puede constituir una alternativa interesante a otros antibióticos que se emplean en el tratamiento de algunas formas de periodontitis.

\section{PALABRAS CLAVE}

Periodontitis crónica, Azitromicina, Estudio doble-ciego.

\footnotetext{
* Profesor Asociado de Periodoncia, Escuela de Graduados, Facultad de Odontología, Universidad de Chile.

** Profesor Asociado de Farmacología, Instituto de Ciencias Biomédicas, Programa de Farmacología Molecular y Clínica, Facultad de Medicina, Universidad de Chile.

*** Cirujano Dentista, Periodoncista, Escuela de Graduados, Facultad de Odontología, Universidad de Chile.

**** Cirujano Dentista, práctica privada.
} 


\section{INTRODUCCIÓN}

Las enfermedades periodontales gingivitis y periodontitis son infecciones de los tejidos de protección e inserción de los dientes. De acuerdo con la denominada Teoría específica de la placa bacteriana enunciada por W.Loesche en 1976 (2) la periodontitis puede considerarse como una infección producida por una proliferación específica de algunas especies bacterianas de la placa bacteriana subgingival, entre las cuales cabe mencionar a bacterias gram negativas anaerobias estrictas como Porphyromonas Gingivalis, Prevotella Intermedia, Fusobacterium nucleatum, Treponema dentícola Campylobacter rectus, Peptoestreptococcus micros, Bacteroides forsythus y algunas facultativas como Actinobacillus actinomycetemcomitans y Eikenella Corrodens $(2,3)$.

Es importante destacar la alta prevalencia de estas enfermedades en la población; algo superior al $75 \%$ en adultos entre los 35-44 años (4). De acuerdo con estudios efectuados en 1996 en nuestro país, la prevalencia fue de un $98.8 \%$ en adultos entre $35-44$ años y de un $100 \%$ en adultos entre 65-74 años (5), lo que señala que las enfermedades periodontales están entre las patologías más prevalentes, por consiguiente constituyen un problema importante de Salud Pública.

El factor etiológico principal de las enfermedades periodontales es la placa dental o placa bacteriana $(2,3,6)$, que corresponde a una acumulación organizada de bacterias sobre la superficie dentaria y puede ser supragingival y subgingival. El crecimiento y maduración de la placa bacteriana supragingival influencia el crecimiento, acumulación y potencial patogénico de la placa subgingival, la que finalmente causa la destrucción del periodonto (7).

En cuanto al tratamiento, la mayoría de las periodontitis pueden ser tratadas con éxito removiendo la placa supra y subgingival mediante el raspado y pulido radicular, siempre asociado a una terapia de mantención continua por parte del profesional y un adecuado control de la placa por parte del paciente (8). Sin embargo, en algunos casos puede ser necesario asociar a la terapia mecánica inespecífica convencional, el uso de antiobióticos que complementan la eficacia de la anterior (9).

Es así como en las últimas décadas se ha demostrado la utilidad de la administración sistémica de algunos antimicrobianos como las tetraciclinas $(10,11)$, el metronidazol (12, 13 y la asociación de metronidazol + amoxicilina $(14,15)$ en el tratamiento de algunas formas de periodontitis.
Las tetraciclinas, además de exhibir un amplio espectro de acción sobre la flora periodontopatógena mencionada $(16,17)$, alcanzan elevadas concentraciones en el surco gingival, incluso superiores a aquellas que se detectan en la sangre $(18,19)$. Además, las tetraciclinas por un mecanismo independiente de su actividad antimicrobiana, inhiben a las colagenasas, algunas de las enzimas encargadas de degradar el colágeno $(20,22)$.

El metronidazol, con amplio espectro de acción sobre la flora anaerobia estricta también alcanza altas concentraciones en el surco gingival, superiores a las concentraciones inhibitorias mínimas de la flora involucrada y ha sido utilizado con éxito en diversas investigaciones $(12,13,23-25)$. Por último la asociación de metronidazol + amoxicilina ha demostrado su eficacia en Periodoncia, incluyendo a pacientes que no han respondido satisfactoriamente a la terapia mecánica convencional asociada a las tetraciclinas $(26,28)$.

La azitromicina es un antibiótico azálido, relacionado estructuralmente con la eritromicina, de la cual difiere por su mayor espectro, mejores características farmacocinéticas y menor toxicidad (29). Estudios efectuados en la última década han demostrado la actividad de la azitromicina sobre las bacterias asociadas al desarrollo de la periodontitis $(30,31)$ lo que plantea la hipótesis que este antibiótico en virtud a su espectro de acción, excelente absorción gastrointestinal y mejor distribución en los tejidos del organismo debe constituir un antibiótico con una potencial aplicación en Periodoncia. Adicionalmente, otros estudios han demostrado interesantes concentraciones de azitromicina en la saliva y en los tejidos periodontales (32, 33) lo que constituye sin duda una característica farmacocinética interesante que es compatible con el elevado volumen de distribución del antibiótico en el organismo. Cabe agregar que las elevados concentraciones del antibiótico en los diversos tejidos se ha atribuido a la capacidad del antibiótico de ser captada por los leucocitos, los cuales posteriormente lo liberan en los sitios de la infección (43).

En consideración a lo anterior, la presente investigación tiene por objetivo evaluar la eficacia de la administración sistémica de azitromicina comparada con el raspado y pulido radicular en un grupo de pacientes con periodontitis crónica.

\section{MATERIAL Y MÉTODO}

La presente investigación corresponde a un estudio prospectivo, controlado, randomizado, doble-ciego en un total de 19 pacientes con periodontitis crónica 
moderada-avanzada, los cuales se atendieron en la clínica de Periodoncia, Escuela de Graduados, Facultad de Odontología, Universidad de Chile.

Los pacientes seleccionados fueron adultos de ambos sexos con el diagnóstico clínico y radiográfico de periodontitis crónica moderada-avanzada del adulto de acuerdo con el siguiente criterio:

1.-Estado de salud general bueno.

2.-Rango de edad entre 38 a 60 años.

3.-Presencia de sacos periodontales de 5 o más milímetros, que sangraron a la exploración y/o supuración ubicados en un mismo maxilar.

4.-Evidencia radiográfica de pérdida de hueso alveolar, superior a un $30 \%$.

Los pacientes con antecedentes de diabetes, embarazo, en etapa de lactancia, y con antecedentes de hipersensibilidad a la azitromicina fueron descartados del estudio. Asimismo, no se incluyeron en la investigación aquellos pacientes que habían recibido antibióticos, antiinflamatorios no esteroidales o enjuagatorios bucales, en los tres últimos meses precedentes como también los fumadores (más de 10 cigarrillos/día)

\section{DISEÑO EXPERIMENTAL}

Cada paciente con el diagnóstico clínico-radiográfico de la afección, fue informado de los beneficios y/o riesgos del estudio para así obtener su consentimiento. Una vez superada esta etapa, se les hizo una instrucción de higiene oral (Técnica de cepillado de Bass y uso de seda dental) y una higienización supragingival de toda la boca, mediante ultrasonido Luego se tomaron impresiones con alginato del maxilar seleccionado y se obtuvieron modelos en yeso donde se confeccionaron canaletas de acrílico que sirvieron como punto de referencia fijo para medir los niveles de inserción relativos y la profundidad de sacos. Posteriormente, se seleccionaron 4 dientes por paciente en un mismo maxilar; dos izquierdos y dos derechos, que tuvieran sacos periodontales $\geq$ a 5 milímetros y que sangraron a la exploración con la sonda Columbia.

En cada diente seleccionado se estudiaron los siguientes parámetros:

1. Indice gingival, de Loe y Silness, en la escala 0, 1, 2, 3.

2. Indice de placa, de Silness y Loe, en la escala 0, 1, 2, 3.

3. Profundidad de sacos. Mediante una sonda periodontal graduada en milímetros (Sonda Columbia) se midió la distancia comprendida entre el fondo del saco periodontal y el margen gingival.

4. Nivel de inserción relativo. Corresponde a la distancia en milímetros desde el fondo del saco periodontal a un punto fijo de referencia ubicado en el borde de la canaleta de acrílico previamente confeccionada.

El día 0, correspondió a aquel día en que los pacientes recibieron la primera atención por parte de los clínicos, en la que bajo anestesia local y mediante el uso de curetas, se efectuó el raspado y pulido radicular en dos de las piezas dentarias elegidas y en aquellas adyacentes, dejando las restantes dos piezas seleccionadas como control, (diseño "split mouth" o boca partida), que permite tener en un mismo paciente dientes raspados y pulidos y otros como control que no reciben este tratamiento.

A continuación y por selección al azar, se entregó a cada paciente un frasco de plástico que incluyó 3 comprimidos de azitromicina (Azitrom ${ }^{\oplus}$ Laboratorio Chile) $500 \mathrm{mg}$ o de placebo; ambos tenían el mismo aspecto exterior para preservar el método dobleciego. Cada frasco de antibiótico o de placebo se identificó solamente por un número conocido por un investigador ajeno al tratamiento clínico de los pacientes. Además de lo anterior, en cada uno de los sujetos incluidos en la investigación se les instruyó para tomar 1 comprimido cada 24 horas durante tres días, y la citación al control de los 30 días y 60 días. El control inicial del día 0 , así como también las mediciones de los días 30 y 60 fueron efectuadas por un mismo investigador, quien ignoraba la naturaleza de los tratamientos recibidos con anterioridad en cada paciente, con el objetivo de preservar la naturaleza doble ciego de la investigación.

Finalmente cabe agregar que aquellos dientes que no recibieron el beneficio del tratamiento convencional fueron tratadas con posterioridad al control de los 60 días de los pacientes.

El número total de la muestra de fue de 19 pacientes de los cuales por selección al azar 10 recibieron azitromicina y 9 tomaron el placebo. Como cada paciente aportó 4 dientes al estudio, el número total de dientes estudiados fueron 76 .

\section{Análisis de los resultados}

En consideración al diseño propuesto, se dispuso de dientes que recibieron uno de los siguientes tratamientos:

1.- Azitromicina.

2.- Azitromicina + raspado y pulido.

3.- Placebo.

4.- Placebo + raspado y pulido. 


\section{Análisis estadístico}

Los resultados que se presentan a continuación corresponden a aquellos que se obtuvieron durante el seguimiento de los pacientes en el control de 30 días. Los resultados finales, incluyendo ambos controles a los 30 y 60 días se reportarán en otra comunicación.

En cada uno de los parámetros estudiados se calcularon los valores medios \pm el error típico (s.e.) de los dientes que recibieron cada uno de los 4 tratamientos descritos, los que se compararon mediante el test " $t$ " de Student. La comparación entre los tratamientos se efectuó mediante el análisis de varianza, seguido del test de Student-Newman.Keuls, cuando los valores " $f$ " revelaron diferencias significativas. Se consideraron significativos todos aquellos valores que tuvieron un valor $\mathrm{p}<0.05$.

\section{RESULTADOS}

Los resultados que se incluyen corresponden a 19 pacientes tratados, de los cuales por selección al azar 10 recibieron azitromicina y 9 tomaron el placebo.

\section{1.- Efectos en el Indice Gingival}

Los efectos de los tratamientos en el Indice Gingival aparecen en la tabla 1 .

\begin{tabular}{|c|c|c|c|c|}
\hline \multicolumn{5}{|c|}{$\begin{array}{c}\text { Tabla l } \\
\text { EFECTOS DE LOS TRATAIMIENTOS EN EL } \\
\text { ÍNDICE GINGIVAL (m } \pm \text { s.e.) }\end{array}$} \\
\hline Tratamiento & Día 0 & Día 30 & "t" & $\mathbf{P}<$ \\
\hline Azitromicina & $2,15 \pm 0,08$ & $1,22 \pm 0,13$ & 6,09 & 0.000 \\
\hline Azitrom. + r.p. & $2,10 \pm 0,07$ & $1,06 \pm 0,15$ & 6,28 & 0.000 \\
\hline Placebo & $2,20 \pm 0,13$ & $1,70 \pm 0,15$ & 2,51 & 0.01 \\
\hline Placebo + r.p. & $2,20 \pm 0,13$ & $1,20 \pm 0,13$ & 5,43 & 0.000 \\
\hline
\end{tabular}

Puede observarse que todos los tratamientos redujeron significativamente este parámetro con diferentes niveles de significación. La comparación entre éstos mediante el análisis de varianza demostró diferencias $(F=3,96 ; p<0.01)$. Posteriormente, el test de Student-Newman.Keuls, para comparaciones múltiples reveló una superioridad de todos los tratamientos sobre el grupo placebo $(\mathrm{p}<0.05)$

\section{2.- Efectos en el Indice de Placa}

Estos resultados, que se expresan en los valores medios \pm el error estándar, aparecen a continuación en la tabla 2.

\begin{tabular}{|c|c|c|c|c|}
\hline \multicolumn{5}{|c|}{$\begin{array}{c}\text { Tabla } 2 \\
\text { EFECTOS DE LOS TRATAMIENTOS EN EL } \\
\text { ÍNDICE DE PLACA (m } \pm \text { s.e.) }\end{array}$} \\
\hline Tratamiento & Día 0 & Día 30 & "t" & $\mathbf{P}<$ \\
\hline Azitromicina & $2,10 \pm 0,14$ & $1,63 \pm 0,11$ & 2,64 & 0,01 \\
\hline Azitrom + r.p. & $1,95 \pm 0,17$ & $1,50 \pm 0,18$ & 1,81 & n.s. \\
\hline Placebo & $2,10 \pm 0.23$ & $1,80 \pm 0.25$ & 0,88 & n.s. \\
\hline Placebo + r.p. & $2,06 \pm 0,21$ & $1,80 \pm 0,24$ & 0.81 & n.s. \\
\hline
\end{tabular}

En la tabla anterior puede apreciarse que sólo el tratamiento con azitromicina redujo significativamente el Indice de Placa $(\mathrm{p}<0.01)$, efecto que no se detectó con el raspado y pulido radicular. y que tampoco apareció cuando el antibiótico se asoció a este último tratamiento.

\section{3.- Efectos en la profundidad de los sacos.}

Estos resultados que se expresan en milímetros y se representan mediante los valores medios \pm s.e, aparecen en la tabla 3.

\section{Tabla 3}

EFECTOS DE LOS TRATAMIENTOS EN LA PROFUNDIDAD DE LOS SACOS (m \pm s.e.)

\begin{tabular}{lcccc}
\hline Tratamiento & Día 0 & Día 30 & "t" & P< \\
\hline Azitromicina & $4,69 \pm 0,15$ & $3,65 \pm 3,40$ & 2,43 & 0.02 \\
\hline Azitrom. + r.p. & $5,05 \pm 0,22$ & $3,32 \pm 0,21$ & 5,68 & 0.000 \\
\hline Placebo & $4,90 \pm 0,32$ & $4,13 \pm 0,32$ & 1,70 & 0.29 \\
\hline Placebo + r.p. & $4,96 \pm 0,29$ & $3,91 \pm 0,24$ & 2,78 & 0,01 \\
\hline r.p. $=$ raspado y pulido & & & \\
\hline
\end{tabular}

Como puede observarse en la Tabla 3, hubo una significativa reducción en la profundidad de los sacos con todos los tratamientos, con la sola excepción del grupo placebo. Sin embargo, la comparación entre aquellos mediante el análisis de varianza no demostró diferencias estadísticamente significativas $(\mathrm{f}=1,34 ; \mathrm{p}$ = no significativo)

\section{4.- Efectos de los tratamientos en el nivel de inserción}

Los efectos de los tratamientos estudiados en el Nivel de inserción, expresados en milímetros \pm s.e. aparecen en la Tabla 4 


\begin{tabular}{|c|c|c|c|c|}
\hline \multicolumn{5}{|c|}{$\begin{array}{l}\text { Tabla } 4 \\
\text { EFECTOS DE LOS TRATAMIENTOS EN EL } \\
\text { NIVEL DE INSERCIÓN (m } \pm \text { s.e.) }\end{array}$} \\
\hline Tratamiento & Día 0 & Día 30 & "t" & $\mathbf{P}<$ \\
\hline Azitromicina & $9,29 \pm 0,45$ & $8,54 \pm 0,54$ & 1,06 & 0.29 \\
\hline Azitrom. + r.p. & $9,27 \pm 0,30$ & $8,01 \pm 2,41$ & 2,87 & 0.01 \\
\hline Placebo & $8,80 \pm 0,42$ & $8,50 \pm 0,43$ & 0,49 & 0.6 \\
\hline Placebo + r.p. & $9,25 \pm 0,52$ & $8,92 \pm 0,52$ & 0,45 & 0.65 \\
\hline
\end{tabular}

Puede apreciarse que hubo una mejoría significativa en el nivel de inserción sólo en aquellos dientes que fueron tratados con el antibiótico asociado al raspado y pulido radicular $(t=2,87) ; \mathrm{p}<0.01$. Sin embargo, la comparación entre los tratamientos mediante el análisis de varianza no demostró diferencias estadísticamente significativas $(f=0,66$ ).

\section{Reacciones adversas}

De la muestra total de 10 pacientes que recibieron azitromicina, sólo cabe mencionar que hubo dos pacientes que reportaron algunas molestias digestivas menores (dolor epigásrico, naúsea), las cuales no fueron causales para suspender el tratamiento. En el grupo placebo, no se detectó la ocurrencia de reacciones adversas.

\section{DISCUSIÓN}

Es ampliamente conocido que las diferentes formas de Periodontitis pueden tratarse efectivamente mediante una correcta remoción de la placa supra y sub-gingival, realizando raspado y pulido radicular asociado a una terapia de mantención continua por parte del profesional y un adecuado control de placa efectuado por el paciente. $(8,35,37)$.

Sin embargo, en algunos pacientes que presentan esta enfermedad, a pesar de haberse efectuado una terapia técnicamente adecuada, se evidencia una continua pérdida de inserción, lo que puede explicarse en parte por una remoción incompleta de los depósitos microbianos, del tártaro subgingival en los dientes involucrados, debido a dificultades inherentes al tratamiento como es la visualización dificultosa por parte del profesional. La falta de éxito en el tratamiento también puede deberse a que las principales especies patógenas involucradas, Actinomyces actinomycetemcomitans, Porphyromonas gingivalis, Prevotella intermedia, Bacteroides forsythus y otras bacterias gram $(-)$, anaerobias o facultativas están provistas de meca- nismos que les permiten invadir el tejido periodontal adyacente a los sitios tratados y reinfectarlos (38), e incluso establecer nichos ecológicos en otros sitios de la cavidad orla como amigdalas, lengua, piso de la boca etc.

Como ya mencionamos, la periodontitis puede considerarse como una infección específica, producida por la proliferación de algunas bacterias de la placa dental subgingival $(2,3)$. Lo anteriormente expuesto ha reforzado el concepto del uso de antimicrobianos en el tratamiento de las periodontitis como un complemento a la terapia mecánica inespecífica convencional (raspado y pulido radicular), y así aumentar la eficacia de la misma En las últimas décadas se ha demostrado la utilidad de la administración sistémica de tetraciclina $(10,11)$; metronidazol $(12,13)$ y la asociación de metronidazol con amoxicilina $(14,15,27)$, en el tratamiento de algunas formas de periodontitis.

La azitromicina, es un antibiótico perteneciente a la familia de los azálidos, relacionado estructuralmente con la eritromicina y que presenta varias ventajas con respecto a ésta última, como mayor espectro de acción, mejores características farmacocinéticas y menor toxicidad $(29,39-42)$. Este antibiótico presenta varias características farmacológicas que revisten particular interés en Periodontología. Por ejemplo, algunos estudios han demostrado que después de su administración oral el antibiótico alcanza altas concentraciones en la saliva y en tejidos periodontales sanos y enfermos $(32,33)$, lo que constituye sin duda una característica farmacocinética interesante que es compatible con el elevado volumen de distribución del antibiótico en el organismo. Adicionalmente, la azitromicina presenta actividad frente a la flora gram negativa anaerobia y facultativa que se asocian al desarrollo de las lesiones periodontales $(30,31)$.

En atención a las características de la azitromicina, esta investigación tuvo por objetivo fundamental evaluar su eficacia en un grupo de pacientes con Periodontitis crónica mediante el análisis de diversos parámetros clínicos que habitualmente se estudian en este tipo de enfermos, analizando los resultados clínicos en controles de 30 y 60 días, y reportando en esta comunicación los primeros disponibles en el control de los 30 días.

Los resultados obtenidos demostraron que todos los tratamientos redujeron el Indice Gingival, siendo superior la eficacia del antibiótico o del raspado y pulido sobre el placebo. Asimismo, en el indice de Placa se demostró que solo la terapia antibiótica mejoró significativamente éste parámetro, un efecto que también se demostró en la profundidad de los sacos periodontales. Por último, en el Nivel de Inserción sólo la terapia antibiótica. asociada al raspa- 
do y pulido radicular mejoró significativamente este parámetro, aunque no hubo diferencias significativas entre los tratamientos.

Como puede apreciarse, la terapia antibiótica redujo significativamente diversos parámetros estudiados, como única medida terapéutica y también cuando se asoció al raspado y pulido radicular, aunque estos efectos también aparecieron en aquellos dientes que recibieron el tratamiento habitual de la afección. Sin embargo, cabe reiterar la superioridad del tratamiento antibiótico en el Indice Gingival e Indice de Placa y asociado al raspado radicular, los efectos sobre la profundidad de los sacos y el nivel de inserción.

En términos generales, puede considerarse que en esta evaluación preliminar efectuada en 19 pacientes y considerando sólo el período control de 30 días, los resultados obtenidos son interesantes, lo que confirmaría la eficacia de un antibiótico que presenta un amplio espectro sobre los periodontopatógenos, y que se concentra en los tejidos periodontales.

Conviene señalar que existe una limitada información referente a la utilización de azitromicina como complemento al raspado y pulido radicular en el tratamiento de la periodontitis, lo que dificulta la comparación de nuestros resultados con aquellos reportados con anterioridad. $\mathrm{Al}$ respecto, cabe mencionar resultados en una muestra inicial de nuestra muestra de pacientes en los cuales se obtuvieron resultados similares a los que se informa en esta oportunidad (45). De la misma manera, en un estudio reciente Smith y col. en una muestra de 46 pacientes con periodontitis crónica, demostraron que una terapia con azitromicina como complemento al raspado y pulido radicular redujo significativamente el porcentaje de sacos profundos, superiores a $5 \mathrm{~mm}$, en comparación con el grupo placebo, y también el promedio en milímetros de la profundidad de los sacos periodontales entre 4 a $5 \mathrm{~mm}$ fue estadísticamente inferior al grupo placebo (46).

En resumen, y atendiendo a las características farmacológicas de la azitromicina, y a los resultados obtenidos tanto clínicos como microbiológicos (44-46) cabe señalar que este antibiótico representa una interesante alternativa a otros antimicrobianos en el tratamiento de algunas formas de periodontitis, de manera que el desarrollo de otras investigaciones en este campo con un mayor número de pacientes y sitios estudiados indudablemente que serán de extremada utilidad para confirmar la eficacia de la azitromicina, como complemento a la terapia mecánica convencional en el tratamiento de alguna formas de periodontitis.

\section{ABSTRACT}

This investigation was performed in order to study the efficacy of systemic azithromycin associated to mechanichal debridement in a sample of patients with chronic periodontitis. The study had a double-blind, prospective and randomized design in a sample of 19 patients with the clinical diagnosis of chronic periodontitis. Clinical measurements were made of the following parameters: Gingival Index, Plaque Index, Bleeding upon probing, Pocket depth and Attachment levels, that were assessed at baseline and at 30 and 60 days.

By random selection and under double-blind conditions 10 patients received azithromicyn ( $500 \mathrm{mg}$ each 24 hours during three days )and 9 received a placebo. At the start of the investigation all patients underwent a full supragingival hygienization treatment with ultrasound, then alginate impressions were taken in order to obtain stone models where acrylic stents were made as fix reference marks to determine pocket depth and attachment levels.

Four teeth were selected from each patient, located in the same maxillar for studying the above parameters days 0,30 and 60 . From that selected teeth, two were scaled and root planed with conventional Gracey curettes and the others remained as controls. Therefore, according with this design, there were 4 possible treatment groups: 1. Azithromycin 2. Azithromycin + scaling and root planing 3 Placebo 4. Placebo + scaling and root planing. All patients were evaluated by the same callibrated investigators under double- blind conditions.

The following results were obtained at 30 days of control: Azithromycin significantly reduced gingival inflammation and pocket depths as compared to its respective baseline values, and when the antibiotic was combined with scaling and root planing, significantly improved attachment levels, however comparison between treatments demonstrated a significant superiority only in the parameter Gingival Index.

This preliminary results obtained at a 30 days period of control suggest that azithromycin associated to scaling and root planing could be regarded as an interesting alternative to other antibiotics that presently are used in the treatment of some forms of periodontitis

\section{KEY WORDS}

Chronic periodontitis - Systemic azithromycin Double blind study 


\section{CORRESPONDENCIA}

Dr. Fernando Gallardo R.

Programa de Farmacología Molecular y Clínica

Instituto de Ciencias Biomédicas

Facultad de Medicina

Universidad de Chile

Casilla 70.000, Correo 7

CHILE

fgallard@machi.med.uchile.clThis investigation was performed in order to study the efficacy of systemic azithromycin associated to mecha

\section{BIBLIOGRAFÍA}

1.International Workshop for a classification of periodontal diseases and conditions. Annals of Periodontol 1999, 4: \# 1 , December

2. Loesche, W. J. The bacterial etiology of periodontal disease: the specific plaque hypothesis. Clinical Dentistry. 1987, 2, chapter 2: 1-10

3. Tanner, A., Maiden, M. F. J., Macuch, P. et al. Microbiota of health, gingivitis and initial periodontitis. J. Clin. Periodontol. 1998, 25: 85-98

4. Organización Mundial de la Salud. Informe de un grupo científico. Epidemiología, etiología y prevención de las periodontopatías. Serie de informes técnicos 621 , Ginebra 1978

5. Gamonal, J. Prevalencia de las enfermedades periodontales y de caries dental en la población de 35-44 y de 6574 años de nivel socioeconómico bajo y medio-bajo de la provincia de Santiago, Región metropolitana y determinación de los recursos humanos necesarios para su tratamiento. Marzo, 1996

6. Haffajee, A. D., Cugini, M. A., Tanner A. et al. Subgingival microbiota in healthy, well-maintained elder and periodontitis subjects. J. Clin Periodontol 1998, 25: 346-53

7. Carranza F.A. Periodontología Clínica de Glickman, Nueva Editorial Interamericana $7^{\text {a }}$ edición, 1994

8. Ramfjord, S. P., Knowles, J. W. et al. Results following three modality of periodontal therapy. J. Periodontol. 1975, 42: 522-6

9. The American Academy of Periodontology. Systemic antibiotics in periodontics. J. Periodontol. 1996, 67: 831-8.

10. Kornman K. S., Karl E. H. The efect of long term low-dose tetracycline therapy on the subgingival microflora in refractory adult periodontitis. J. Periodontol. 1982, 53: 604-10

11. Scopp E. W., Froum S. J., Sullivan M. Tetracycline: clinical study to determine its effectiveness as a long-term adjuvant. J. Periodontol. 1980, 51: 328-30

12. Noyan, U., Yilmaz, S., Kuru, B., et al. A clinical and microbiological evaluation of systemic and local metronidazole in adult periodontitis patients. J. Clin. Periodontol. 1997, 24: 158-65

13. Winkel, E. G., Van Winkelhoff, A. J., Timmerman, F., et al. Effects of metronidazole in patiens with refractory periodontitis associated with Bacteroides forsythus. J. Clin. Periodontol. 1997, 24: 573-9

14. Flemming. T. F., Milián, E., Karch, H. \& Klaiber, B. Differential clinical treatment outcome after sistemics metronidazole and amoxicillin in patients harbouring A.a. and/or P.g. J. Clin. Periodontol 1998, 25: 380-7

15. Van Winkelhoff A.J., Tijhof C.LJ., de Graaf J. Microbiological and clinical results of metronidazole plus amoxicillin therapy in Actinobacillus actinomycetemcomitans-associated periodontitis. J.Periodontol 1992, 63: 52-7

16. Walker C.B. Antibiotic susceptibilities of periodontal bacteria: in vitro susceptibilities to eight antimicrobial agents. J.Periodontol 1985, 56 (Suppl) 67-74

17. Baker P.J., Evans R.T.,Slots J, Genco R.J. Susceptibility of human oral anaerobic bacteria to antibiotic suitable for topical use. J.Clin Periodontol 12: 1985, 201-8

18. Gordon J.M.,Walker C., Murphy J.C. et al. Concentration of tetracycline in human gingival fluid after single doses. J.Clin Periodontol 1981, 8: 117-21

19. Pascale D., Gordon J., Lamster I., et al. Concentration of doxycycline in human gingival fluid. J.Clin Periodontol 1986, 13: 841-4

20. Golub L.M., Ramamurthy N., Mc Namara T.F. et al. Tetracycline inhibit tissue collagenase activity. A new mechanism in the treatment of periodontal disease. J.Periodontal Res 1984, 19: 651-5

21. Golub L.M., Lee H.M.,Lehrer G. et al. Minocycline reduces gingival collagenolitic activity during diabetes. Preliminary observations and a proposed new mechanism of action. J.Periodontal Res 1983, 18: 516-26

22. Golub L.M., Wolff L.S., Lee H.M et al. Further evidence that tetracyclines inhibits collagenase activity in human crevicular fluid and from other mammalian sources. J.Periodontal Res 1985, 20: 12-23 
23. Loesche W.J., Syed S.A., Morrison E.C., et al. Metronidazole in periodontitis l. Clinical and bacteriologic results after 15 to 30 weeks. J.Periiodontol 1984, 55: 325-35

24. Loesche W.J., Schmidt E., Smith B.A. et al. Metronidazole therapy for periodontitis. J.Periodontol 1987, 22: 224-6

25. Saxen L., Asikainen S. Metronidazole in the treatment of localized juvenile periodontitis. J.Clin Periodontol 1993, 20: 166-71

26. Van Winkelhoff A.J., Tijhof C.LJ., de Graaf J. Microbiological and clinical results of metronidazole plus amoxicillin therapy in Actinobacillus actinomycetemcomitans-associated periodontitis. J. Periodontol 1992, 63: 52-7

27. López N., Gamonal J.A. Effects of metronidazole plus amoxycillin in progressive untreated adult periodontitis: report of a single l-week course after 2 and 4 month. J.Periodontol 1998, 69: 1291-8

28. Winkel, E.G., Van Winkelhoff A.J., Van der Velden U. Additional clinical and microbiological effects of amoxicillin and metronidazole after initial periodontal therapy. J.Clin Periodontol 1998, 25: 857-64

29 Peters D., Fridel H., Mc Tavish D. Azithromycin: a review of its antimicrobial activity, pharmacokinetic properties and clinical efficacy. Drugs 1992, 44: 750-99

30. Pajukanta, R. In vitro antimicrobial susceptibility of porphyromonas gingivalis to azithromycin, A novel macrolide. Oral Microbiol.Immunol. 1993, 8: 325-6

31. Pajukanta R, Asikainen S, Saarela M, et al. In vitro activity of azithromycin compared with that of erithromycin against Actinobacillus actinomycetemcomitans. Antimicrob Agents Chemother 1992, 36: 1241-3.

32. Blandizi C., Malizia T., Lupetti A. et al. Periodontal tissue disposition of azithromycin in patiens affected by chronic inflammatory periodontal disease. J. Periodontol. 1999, 70: 960-6

33. Malizia T., Tejada M.R., Ghelardi E. Et al. Periodontal tissue disposition of azithromycin. J. Periodontol 1997, 68: 1206-9

34. Glaude, R. P., Bright, G. M., Isaacson, R. E. et al. In vitro and in vivo uptake of Azithromycin (CP-62,993) by phagocityc cells; possible mechanism of delivery and release at sites of infection. Antimicrob. Agents Chemother. 1989, 33: 277-82
35. Lindhe J., Nyman S. The effect of plaque control and surgical pocket elimination on the establishment and maintenance of periodontal health. A longitudinal study of periodontal therapy in cases of advanced disease. J.Clin Periodontol 1975, 2: 67-9

36. Badersten A., Nilveus R., Egelberg J. Effects of nonsurgical periodontal therapy.1.Moderately advanced periodontitis. J.Clin Periodontol 1981, 8: 57-72

37. Pihlstrom B., McHugh R., Oliphant T., Ortiz-Campos C. Comparison of surgical and nonsurgical treatment of periodontal disease. A review of current studies and adittional results after 6 years. J.Clin Periodontol 1983, 10: 524-41

38. Saglie, F. R., Marfany, A.,Camargo, P. Intragingival ocurrence of Actinobacillus actinomycetemcomitans and Bacteroides gingivalis in active destructive periodontal lesions. J. Periodontol. 1988, 59: 259-65

39. Sefton, A. M., Maskell, J. P., Beighton, et al. Azithromycin in the treatment of periodontal disease. Effect on microbial flora. J.Clinical Periodontology. 1996, 23: 998-1003

40. Hoepelman IM, Schneider MME. Azithromycin: The first of the tissue-selective azalides. Int. J Antimicrob Agents 1995, 5: 145-67

41. Neu, H. Clinical microbiology of Azithromycin. Am. J. Med., 1991, 91: (suppl 3 A): 12S-18S

42. Schlossberg, D. Azithromycin and Clarithromycin. Med. Clin. North Am. 1995, 79: 803-15

43. Glaude, R. P., Bright, G. M., Isaacson, R. E., Newborg, M. E. In vitro and in vivo uptake of Azithromycin (CP-62,993) by phagocityc cells; possible mechanism of delivery and release at sites of infection. Antimicrob. Agents Chemother. 1989, 33: 277- 82

44. Sefton, A.M., Maskell, J.P., Beighton, D., et al. Azithromycin in the treatment of periodontal disease. Effect on microbial flora. J.Clin. Periodontol. 1996, 23: 998-1003

45. Rioseco M.P. Vargas C.L. Azitromicina en el tratamiento de la periodontitis del adulto. Trabajo de investigación para optar al título de Cirujano-Dentista. Universidad de Chile, Facultad de Odontología, 1999

46. Smith S.R., Foyle D.M., Daniels J. et al. A double-blind placebo-controlled trial of azithromycin as an adjunct to non-surgical treatment of periodontitis in adults: clinical results. J.Clin Periodontol 2002, 29: 54-61 